\title{
Dilute Solution Properties of Cyclized Polybutadiene
}

\author{
Kazuhiko YAmamoto, Nobuo BeSSHo, Toru SHIIBASHI, \\ and Etsuji MAEKAWA \\ Tokyo Research Laboratory, Japan Synthetic Rubber Co., Ltd., \\ 7569 Ikuta, Tama-ku, Kawasaki, Kanagawa 214, Japan.
}

(Received September 24, 1980)

\begin{abstract}
Light scattering and viscosity measurements on dilute solutions of cyclized cis1,4-polybutadiene polymers (CBR) with different degrees of cyclization $(D C)$ and their fractions are reported in this paper. The data were obtained in both cyclohexane and mixed theta solvents at $30.0^{\circ} \mathrm{C}$. The curves of the intrinsic viscosity $[\eta]_{0}$ in mixed theta solvents $v s$. the weight average molecular weight $M_{w}$ for CBR with $D C$ over $30 \%$ exhibited two distinct regions with slopes of 0.5 and less. Thus, the exponent in the viscosity equation is the same as that for the original uncyclized samples at $M_{w}$ below the critical point $M_{w c}$ and decrease with increasing $D C$ at $M_{w}$ above $M_{w c}$. The $M_{w c}$ shifts to lower $M_{w}$ as $D C$ is increased. The relation of the molecular structure of CBR with $D C$ is discussed. The results led to the conclusions that 1) at $D C$ below $25 \%$, the molecules are linear in the overall structure, but locally contain short intramolecular cyclic structures, e.g., mono- and/or bicyclic structures, 2) at $D C$ between 25 and $60 \%$, the molecules are randomly branched, and 3) at $D C$ over $80 \%$, the molecules have probably very compact structure formed by internal crosslinks.

KEY WORDS Cyclized Polybutadiene / Degree of Cyclization / Intrinsic

Viscosity / Weight Average Molecular Weight / Mean-Square Radius of Gyration / Mixed Theta Solvent / Dilute Solution /
\end{abstract}

The cyclized cis-1,4-polybutadiene (CBR) produced by Japan Synthetic Rubber Co., Ltd. shows characteristic properties of a photoresists, as has been reported elsewhere. ${ }^{1}$ A variety of solution properties of cyclized polydienes, especially cylized polyisoprene, have already been studied by a group of Czechoslovak Academy of Sciences, ${ }^{2-6}$ who found that the dependence of the limiting viscosity number and the GPC elution volume on the molecular weight is the same for both cyclo- and cyclized polydienes and that the structures of these two polydienes are very different from those of linear polydienes. Thus, the macromolecules of cyclo- and cyclized polydienes are compact and spherical in shape with extraordinarily small dimensions.

In this work, we have examined systematically the dilute solution properties of CBR with cyclization degrees from 0 to $80 \%$ in both good and theta solvents. The weight-average molecular weights, the mean-square radii of gyration, and the intrinsic viscosities of these CBR were determined by light scattering and viscosity measurements. On the basis of the experimental data obtained, the relation between the intramolecular structure of CBR and the degree of cyclization is discussed.

\section{EXPERIMENTAL}

\section{Materials}

Five CBR samples with different cyclization degrees were used in the present work. They were prepared by cyclization of a butadiene polymer, JSR BR01 from our production plant. The details of the cyclization reaction were reported in a previous paper. ${ }^{1}$ The conditions used for our cyclization reactions of the butadiene polymer were quite different from those employed by Bohackova et $a l .{ }^{4}$ Several characteristic parameters of our samples are listed in Table I. The polydispersity of the samples increased considerably with increasing $D C$. More specifically, the number-average molecular weight $M_{n}$ varied little with $D C$, while the weight-average molecular weight $M_{w}$ increased considerably as $D C$ is increased. 
Table I. Characteristic properties of CBR

\begin{tabular}{rrccc}
\hline Sample & DC $(\%)^{\mathrm{a}}$ & {$[\eta] / \mathrm{dl} \mathrm{g}^{-1 \mathrm{~b}}$} & $M_{w} \times 10^{-4 \mathrm{c}}$ & $M_{n} \times 10^{-4 \mathrm{~d}}$ \\
\hline CB-A & 9 & 1.71 & 59.6 & 10.5 \\
-B & 31 & 1.32 & 83.3 & 11.5 \\
-C & 46 & 1.02 & 103.6 & 10.0 \\
-D & 63 & 0.58 & 115.2 & 9.9 \\
-E & 81 & 0.31 & 104.4 & 9.9 \\
\hline
\end{tabular}

a From NMR spectra.

b In cyclohexane at $30^{\circ} \mathrm{C}$.

c By light scattering (cyclohexane, $30^{\circ} \mathrm{C}$ ).

${ }^{d}$ By osmotic pressure (toluene, $37^{\circ} \mathrm{C}$ ).

\section{Fractionation}

Each sample was fractionated by precipitation, using a column constructed at our laboratory. ${ }^{7}$ Elution of the polymer was made with benzene as the solvent and methyl alcohol as the nonsolvent, in such a way that the solvent composition in the mixture increased exponentially as the elution proceeded. Each sample was separated into about 15 fractions. Several fractions having suitable molecular weights were chosen for the present study.

\section{$N M R$}

The cyclization degree of each CBR sample was evaluated by means of ${ }^{1} \mathrm{H}-\mathrm{NMR}$. The measurements were carried out at room temperature using a JEOL $4 \mathrm{H}-100$ spectrometer. A test solution of $10 \%$ polymer concentration was prepared in tetrachloroethylene with tetramethylsilane added as the internal standard.

\section{Theta Solvents}

We could not find for CBR a single (i.e., pure) theta solvent suitable for dilute solution measurements. We found that a mixed theta solvent for CBR could be obtained by mixing cyclohexane $\left(n_{436 \mathrm{~nm}}^{30}=1.430-\right.$ a good solvent $)$ with dioxane $\left(n_{436 \mathrm{~nm}}^{30}=1.427-\right.$ a poor solvent $)$. The important reason for this selection was that the refractive indices of these solvents are almost identical. The weight-average molecular weight $M_{w}$, the second virial coefficient $A_{2}$, and the mean-square radius of gyration $\left\langle s^{2}\right\rangle_{z}$ could therefore be determined by conventional light scattering measurement. The composition of the mixed theta solvent at $30^{\circ} \mathrm{C}$ was determined as a function of the cyclization degree as described below.

\section{Viscosity}

Test solutions of several different concentrations were prepared in cyclohexane and in mixed theta solvents. Viscosity measurements were made at $30.0 \pm 0.1^{\circ} \mathrm{C}$, using a capillary viscometer of the Ubbelohde dilution type. The kinematic energy and end-effect corrections were negligible.

\section{Light Scattering}

A Fica 50 automatic light scattering photometer was used, and the measurement was made over an angular range from 30 to $150^{\circ}$ at $30.0 \pm 0.1^{\circ} \mathrm{C}$. Vertically polarized light of wavelength $436 \mathrm{~nm}$ was used as the incident beam. Test solutions of different çoncentrations were made up from a $0.2 \%$ stock solution prepared by dissolving a given fraction in cyclohexane or in a mixed theta solvent. Each of these was filtered through a $0.45 \mu \mathrm{m}$ Millipore filter and directly poured into a light scattering cell just before measurement. The specific refractive index increment of a given system was determined by a modified differential refractometer of the Schulz type. ${ }^{8}$

\section{RESULTS AND DISCUSSION}

\section{$N M R$}

The NMR spectra of the raw BR and the sample CB-D are illustrated in Figure 1. As seen from the figure, the intensity of the peak at $5.3 \mathrm{ppm}$ decreases with cyclization and a new broad peak appears at near $1.5 \mathrm{ppm}$. From the area $A$ for the proton $-\mathrm{CH}=$ and the area $B$ for the total saturated pro-

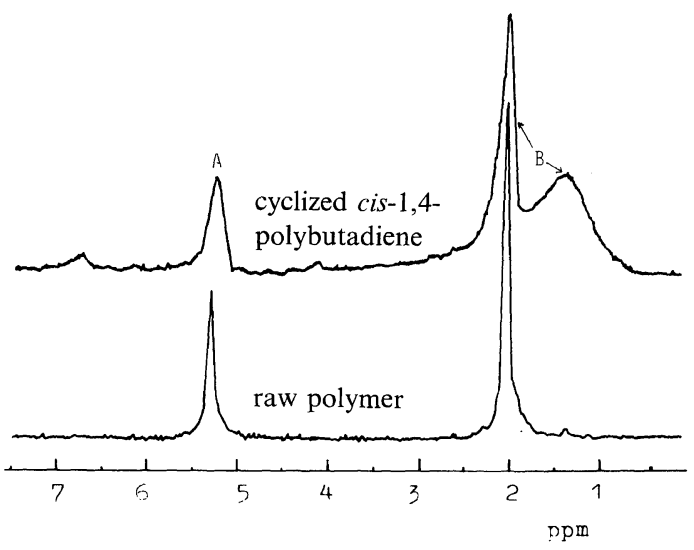

Figure 1. NMR spectra for cyclized polybutadien and its starting polymer. 
Table II. Composition of mixed theta solvents for CBR

\begin{tabular}{cc}
\hline Sample & Cyclohexane/Dioxane (by volume) \\
\hline CB-A & $12 / 88$ \\
-B & $17 / 83$ \\
-C & $21 / 79$ \\
-D & $30 / 70$ \\
-E & $39 / 61$ \\
\hline
\end{tabular}

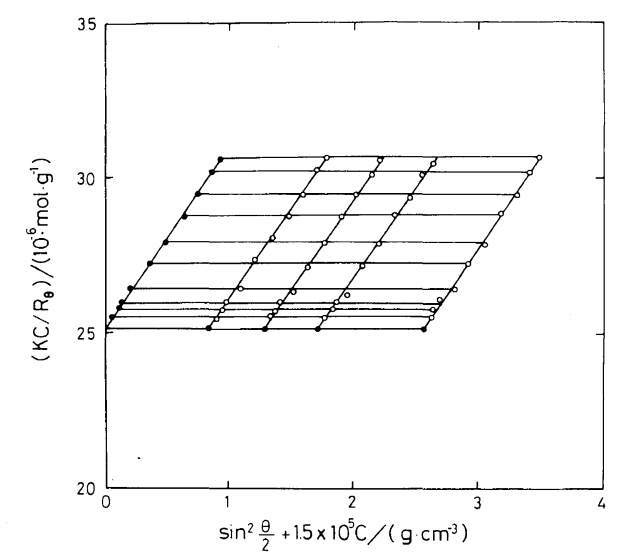

Figure 2. Zimm plot for $\mathrm{CB}-\mathrm{D}-\mathrm{f} 13$ in mixed theta solvents at $30^{\circ} \mathrm{C}$.

tons, the degree of cyclization $(D C$-strictly apparent $D C$ ) was calculated using the following relation,

$$
D C(\%)=\left(1-\frac{3 A}{A+B}\right) \times 100
$$

\section{Composition of Mixed Theta Solvents}

In order to determine the cyclohexane-dioxane ratio of the mixed theta solvent at $30^{\circ} \mathrm{C}$ (theta temperature) as a function of $D C$, light scattering measurements were carried out on the whole CBR polymers. Thus, $A_{2}$ for the sample CB-D vanished at $30^{\circ} \mathrm{C}$ when the volume ratio of cyclohexane to dioxane was 30/70. It was found from NMR data for fractionated CBR that $D C$ of the low and high end fractions were somewhat different (about several per cent). However, their $A_{2}$ were nearly equal to zero under the same solvent conditions. In a similar way, the solvent composition leading to the theta at $30^{\circ} \mathrm{C}$ for all other CBR were finally determined as given in Table II.

\section{Light Scattering and Viscosity}

The customary extrapolations to zero concentration and scattering angle on the Zimm plot ${ }^{9}$ for CB-D-f 13 in the mixed theta solvent are illustrated in Figure 2. Both angular and concentration extrapolations are practically linear. Similar Zimm plots were obtained for all other samples. The values of $M_{w}, A_{2}$, and $\left\langle s^{2}\right\rangle_{z}$ determined for each fraction are summarized in Table III.

For a given polymer, the same $M_{w}$ should be obtained regardless of the solvnt used. Figure 3 shows verification of the identity of two series of $M_{w}$, one determined in cyclohexane and the other in theta solvents.

Figure 4 represents the Mark-HouwinkSakurada plots of $\log [\eta]_{0}$ against $\log M_{w}$ for the fractions in mixed theta solvents at $30^{\circ} \mathrm{C}$. The plots are represented by the following equations,

$$
\begin{array}{ll}
{[\eta]_{0}=K_{0} M_{w}^{0.5}} & \left(\text { below } M_{w_{\mathrm{c}}}\right. \text { ) } \\
{[\eta]_{0}=K_{0}{ }^{\prime} M_{w}{ }^{a}} & \left(\text { above } M_{w_{\mathrm{c}}}\right)
\end{array}
$$

where $K_{0}, K_{0}{ }^{\prime}$, and $a$ are constants independent of $M_{w}$. The data points for the series CB-A fall on the straight line represented by eq 2 . The plots for other series with $D C$ over $30 \%$ begin to deviate from straight lines at a certain $M_{w}$ (this is defined as the critical molecular weight $M_{w_{c}}$ ) and, in each case, consists of two regions, one with a slope of 0.5 and other with a slope of $a$ which is smaller than 0.5 for higher $D C$. Moreover, $M_{w_{\mathrm{c}}}$ shifts to lower $M_{w}$ as $D C$ is increased. The fact that the data are expressed by eq 2 suggests that the molecule behaves hydrodynamically like a linear polymer. This does not always mean that the molecule is literally linear, but implies that, in the range of low $D C$, cyclization occurs in such a way that the molecule has no branches. The viscosity $v s$. molecular weight relations in cyclohexane at $30^{\circ} \mathrm{C}$, though not given here, are represented by equations similar to eq 2 and $2^{\prime}$ with larger exponents.

Figure 5 shows double-logarithmic plots of $\left\langle s^{2}\right\rangle_{0 z}^{1 / 2}$ against $M_{w}$ in mixed theta solvents at $30^{\circ} \mathrm{C}$. We see that the dependence of $\left\langle s^{2}\right\rangle_{0 z}^{1 / 2}$ on $M_{w}$ resembles that of $[\eta]_{0}$, but shows no cirtical molecular weight. The data points for the series CB-A and those for other series are respectively represented by the equations,

$$
\begin{array}{ll}
\left\langle s^{2}\right\rangle_{0 z}^{1 / 2}=k_{0} M_{w}^{0.5} & (\text { for the series CB-A) } \\
\left\langle s^{2}\right\rangle_{0 z}^{1 / 2}=k_{0}{ }^{\prime} M_{w}{ }^{b} & \text { (for other series) }
\end{array}
$$


Table III. Results of viscosity and light scattering measurements ${ }^{\mathrm{a}}$

\begin{tabular}{|c|c|c|c|c|c|c|c|c|}
\hline \multirow{2}{*}{\multicolumn{2}{|c|}{ Franction }} & \multicolumn{3}{|c|}{ Mixed theta solvents } & \multicolumn{4}{|c|}{ Cyclohexane } \\
\hline & & {$[\eta]_{0}$} & $M_{w} \times 10^{-4}$ & $\left\langle s^{2}\right\rangle_{0 z}^{1 / 2} \times 10^{10}$ & {$[\eta]$} & $M_{w} \times 10^{-4}$ & $A_{2} \times 10^{4}$ & $\left\langle s^{2}\right\rangle^{1 / 2} \times 10^{10}$ \\
\hline \multirow{5}{*}{ CB-A } & 9 & 0.59 & 10.8 & 146 & 0.79 & 9.5 & 10.8 & 170 \\
\hline & 10 & 0.71 & 16.1 & 170 & 1.09 & 14.9 & 10.4 & 196 \\
\hline & 11 & 0.88 & 20.4 & 210 & 1.40 & 19.6 & 8.65 & 256 \\
\hline & 12 & 1.10 & 36.2 & 255 & 1.82 & 35.9 & 8.24 & 336 \\
\hline & 13 & 1.45 & 51.8 & 311 & 2.75 & 58.7 & 6.30 & 398 \\
\hline \multirow[t]{8}{*}{$-B$} & 7 & 0.35 & 6.2 & - & 0.50 & 6.7 & 9.13 & 130 \\
\hline & 8 & 0.41 & 8.7 & - & 0.65 & 8.9 & 7.87 & 150 \\
\hline & 9 & 0.45 & 11.8 & 146 & 0.73 & 11.7 & 7.08 & 165 \\
\hline & 10 & 0.55 & 17.2 & 171 & 0.88 & 17.2 & 5.70 & 230 \\
\hline & 11 & 0.60 & 20.5 & 175 & 1.08 & 21.4 & 5.65 & 240 \\
\hline & 12 & 0.70 & 29.9 & 201 & 1.48 & 31.5 & 5.85 & 270 \\
\hline & 13 & 0.89 & 55.0 & 238 & 1.92 & 61.0 & 3.05 & 390 \\
\hline & 14 & 1.14 & 116 & 324 & 2.54 & 121 & 2.15 & 570 \\
\hline \multirow[t]{7}{*}{$-\mathrm{C}$} & 7 & 0.24 & 4.8 & - & 0.34 & 5.3 & 7.09 & - \\
\hline & 9 & 0.28 & 7.4 & - & 0.44 & 8.2 & 6.31 & 122 \\
\hline & 10 & 0.33 & 10.1 & 125 & 0.52 & 9.9 & 6.55 & 150 \\
\hline & 11 & 0.34 & 12.2 & 147 & 0.59 & 12.8 & 5.26 & 154 \\
\hline & 12 & 0.38 & 17.0 & 150 & 0.67 & 17.8 & 3.85 & 184 \\
\hline & 13 & 0.41 & 23.0 & 150 & 0.77 & 23.3 & 4.00 & 218 \\
\hline & 14 & 0.47 & 34.6 & 182 & 0.97 & 39.5 & 2.62 & 266 \\
\hline \multirow[t]{6}{*}{$-\mathrm{D}$} & 5 & 0.18 & 3.6 & - & 0.22 & 3.3 & 3.75 & - \\
\hline & 7 & 0.21 & 5.3 & - & 0.28 & 5.3 & 3.74 & - \\
\hline & 9 & 0.25 & 10.8 & 115 & 0.32 & 10.3 & 3.98 & 129 \\
\hline & 11 & 0.28 & 18.7 & 135 & 0.42 & 17.3 & 2.98 & 179 \\
\hline & 13 & 0.33 & 39.7 & 160 & 0.54 & 39.1 & 1.70 & 264 \\
\hline & 15 & 0.39 & 110 & 221 & 0.72 & 115 & 1.27 & 366 \\
\hline \multirow[t]{5}{*}{$-E$} & 7 & 0.17 & 5.5 & - & 0.22 & 5.7 & 1.89 & - \\
\hline & 9 & 0.19 & 12.9 & 110 & 0.26 & 10.6 & 1.44 & 120 \\
\hline & 11 & 0.20 & 23.5 & 116 & 0.28 & 20.4 & 1.35 & 155 \\
\hline & 13 & 0.21 & 51.0 & 151 & 0.32 & 49.0 & 0.95 & 260 \\
\hline & 15 & 0.22 & 86.0 & 174 & 0.37 & 82.0 & 0.74 & 310 \\
\hline
\end{tabular}

${ }^{\mathrm{a}}[\eta]_{0}$ and $[\eta], \mathrm{dl} \mathrm{g}^{-1} ;\left\langle s^{2}\right\rangle_{0 z}^{1 / 2}$ and $\left\langle s^{2}\right\rangle^{1 / 2}, \mathrm{~m} ; A_{2}, \mathrm{~mol} \mathrm{~cm} \mathrm{~g}^{-1}$.

where $k_{0}, k_{0}{ }^{\prime}$, and $b$ are constants independent of $M_{w}$. The exponent $b$ is also seen to decrease with increasing $D C$.

The above results indicate that the molecular structures of CBR in the regions below and above $M_{w_{c}}$ are quite different from each other. In particular, the molecule above $M_{w_{c}}$ behaves like a branched polymer. This behavior is discussed in detail below.

\section{Molecular structures at $M_{w}$ below $M_{w_{c}}$}

The relation between $K_{0}$ and $D C$ is shown in
Figure 6. It is seen that with increasing $D C, K_{0}$ initially decreases monotonically and appears to be constant for $D C$ above $50 \%$. The mechanism of cyclization and the structure of the product are fully understood for $c i s-\mathrm{BR}$. However, the formation of a six-membered ring (cyclic structure) which is much the same as that in cyclized polyisoprene appears to have been established. ${ }^{10}$. The behavior of $K_{0}$ for $D C$ below $50 \%$ may be explained by a theory analogous to that known for short branching in polyethylene. ${ }^{11}$ Thus, the formation of a short cyclic structure like a mono- and/or bicyclic structure does 


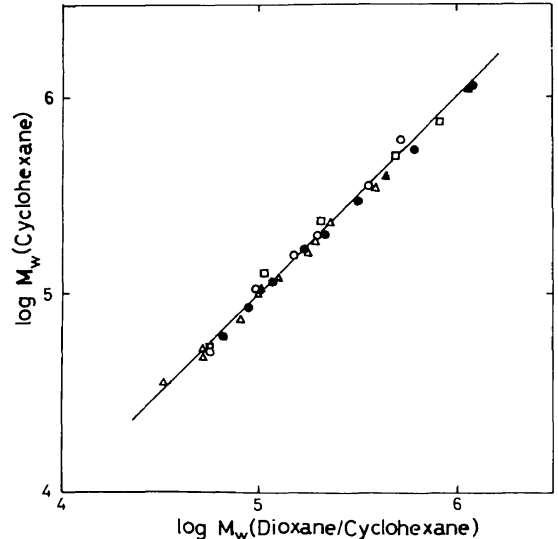

Figure 3. Experimental verification of the $M_{w}$ values determined in cyclohexane and in mixed theta solvents: O, CB-A;, , CB-B; $\triangle$, CB-C; $\boldsymbol{\Delta}$, CB-D; $\square$, CB-E.

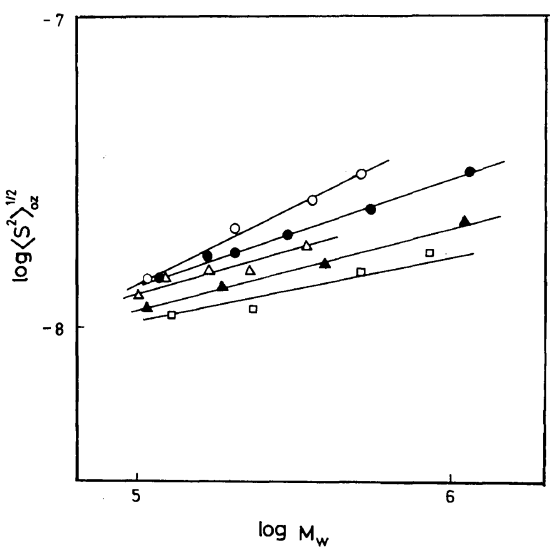

Figure 5. Double logarithmic plots of $\left\langle s^{2}\right\rangle_{0 z}^{1 / 2}$ against $M_{w}$ for each CBR. Symbols are the same as in Figure 3.

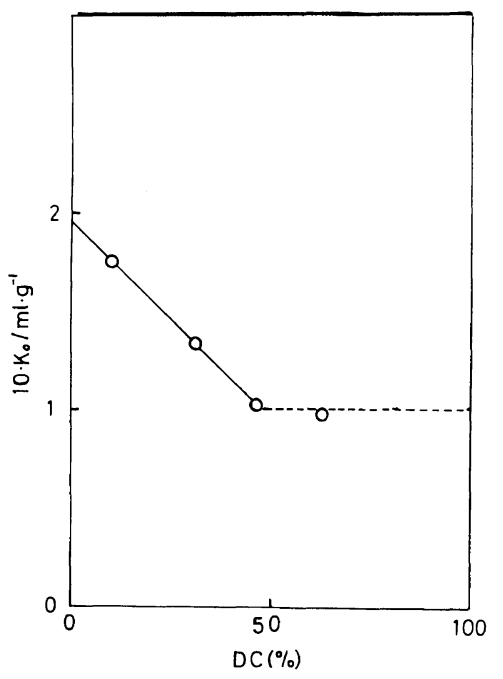

Figure 6. Dependence of $K_{0}$ values on cyclization degree for CBR.

to define three dimensionless parameters $G, g$, and $v$ by,

$$
\begin{aligned}
& G=[\eta]_{0 b} /[\eta]_{0 l} \\
& g=\left\langle s^{2}\right\rangle_{0 b} /\left\langle s^{2}\right\rangle_{0 l} \\
& G=g^{v}
\end{aligned}
$$

where the subscripts $b$ and $l$ indicate branched and linear polymers, respectively. The exponent $v$ is roughly equal to 0.5 in most cases. ${ }^{12}$ Table IV gives the theoretical values of the parameters $G, g$, and $v$ 
Table IV. Theoretical viscosity relations for various molecular structures

\begin{tabular}{llc}
\hline $\begin{array}{c}\text { Type of } \\
\text { structure }\end{array}$ & \multicolumn{1}{c}{ Relation } & References \\
\hline $\begin{array}{l}\text { Branched } \\
\text { Star }\end{array}$ & $G \propto g^{0.5}$ & 13 \\
Comb & $G \propto g^{0.5}$ (low branching) & 14 \\
Random & $G \propto g^{1.5}$ (high branching) & \\
& $G \propto g^{0.5-0.6}, g \propto M^{-0.5}$, & 12 \\
Ring & {$[\eta]_{0} \propto M^{0.2 ~ 0.25}$} & \\
Single & $G \propto g^{0.633}$ & 16 \\
\hline
\end{tabular}

for various types of branched and ring polymers.

In evaluating $G$ and $g$ for CBR, $[\eta]_{0 b}$ and $\left\langle s^{2}\right\rangle_{0 b}$ in eq 4 and 5 should be the intrinsic viscosity and the mean-square radius of gyration of a real CBR sample in the mixed theta solvent, while $[\eta]_{o l}$ and $\left\langle s^{2}\right\rangle_{0 l}$ should represent these values of a hypothetical linear CBR having the same $M_{w}$ as the real CBR. The $[\eta]_{0 l}$ for a series of samples with a fixed $D C$ can be estimated by extrapolating the $[\eta]_{0}$ versus $M_{w}$ relationship for low $M_{w}$ samples to the region of high $M_{w}$. The $D C$ dependence of $K_{0}$ shown in Fig. 6 may be used for this purpose. On the other hand, $k_{0}$ in eq 3 could not be experimentally determined except for the series CB-A. Therefore, we estimate $\left\langle s^{2}\right\rangle_{0 l}$ from $[\eta]_{0 l}$ by using the familiar Flory-Fox relation,

$$
[\eta]_{0 l}=6^{3 / 2} \Phi_{0} \frac{\left\langle s^{2}\right\rangle_{0 l}^{3 / 2}}{M}
$$

where $\Phi_{0}$ is a constant which is the same for all flexible linear polymes under the theta condition. The $\Phi_{0}$ for CB-A was estimated from the experimental values of $[\eta]_{0 l}$ and $\left\langle s^{2}\right\rangle_{0 l}$ to be $1.76 \times 10^{23}$ $\mathrm{mol}^{-1}$. This value is somewhat samaller than the typical theoretical value, but the deviation may be attributed to the molecular weight distributions in
Table V. Structural parameters estimated of CBR

\begin{tabular}{ccccccc}
\hline Sample & $M_{w} \times 10^{-4}$ & $G$ & $g$ & $v$ & $\lambda M$ & $\lambda^{-1} \times 10^{4}$ \\
\hline CB-B & 51.0 & 0.92 & 0.80 & 0.39 & 2.8 & 18 \\
& 116 & 0.75 & 0.65 & 0.67 & 6.8 & 17 \\
-C & 23.3 & 0.87 & 0.82 & 0.68 & 2.6 & 9.0 \\
& 39.5 & 0.81 & 0.71 & 0.60 & 5.0 & 7.9 \\
& & & & & & \\
-D & 19.2 & 0.67 & 0.70 & 1.1 & 5.0 & 3.8 \\
& 44.0 & 0.55 & 0.45 & 0.74 & 20 & 2.2 \\
& 115 & 0.39 & 0.40 & 1.0 & 28 & 4.1 \\
-E & 23.5 & 0.42 & 0.49 & 1.2 & 15 & 1.6 \\
& 51.0 & 0.30 & 0.39 & 1.3 & 30 & 1.7 \\
& 86.0 & 0.24 & 0.31 & 1.2 & 54 & 1.6 \\
\hline
\end{tabular}

the present samples. ${ }^{15}$ In fact, $\left\langle s^{2}\right\rangle_{0 z}$ calculated for CB-B-f 9 below $M_{w}$ was in very good agreement with the measured one. The values of $G, g$, and $v$ thus obtained by eq 4,5 , and 6 are shown in Table $\mathrm{V}$. It can be seen that 1) $G$ and $g$ decrease with increasing $M_{w}$ at constant $D C$ and that they decrease with increasing $D C$ at constant $M_{w}$, and 2) $v$ increases as $D C$ is increased. Thus, the shrinkage of chain dimensions should be attributed to chain branching due either to intramolecular reaction or to crosslinking within the molecule.

As shown in Table I, when cyclization is made on the same unfractionated BR01, $M_{n}$ remains essentially unchanged, while $M_{w}$ increases considerably with increasing $D C$. We made a check of this phenomenon using two fractionated samples of BR01. The results are shown in Table VI, where $M_{n}$ of CBR is almost the same as that of the original BR and $M_{w}$ increases by a factor of 2 or 3 . Thus, we can conclude that the cyclization reaction is accompanied by polymer chain scission and recombination. Hence, it may be appropriate to assume a randomly branched form for the molecular struc-

Table VI. Change in molecular weight by cyclization for BR fractions

\begin{tabular}{ccccccccc}
\hline & \multicolumn{3}{c}{ BR } & & \multicolumn{3}{c}{ CBR } \\
\cline { 2 - 3 } \cline { 6 - 8 } & $M_{n} \times 10^{-4}$ & $M_{w} \times 10^{-4}$ & $M_{w} / M_{n}$ & & $M_{n} \times 10^{-4}$ & $M_{w} \times 10^{-4}$ & $M_{w} / M_{n}$ & DC $(\%)$ \\
\hline F1 & 2.4 & 2.6 & 1.1 & & 2.6 & 5.7 & 2.2 & 52 \\
F2 & 4.7 & 5.2 & 1.1 & & 5.0 & 14.1 & 2.8 & 60 \\
\hline
\end{tabular}


ture of CBR.

For a randomly branched molecule, the theory ${ }^{12}$ gives,

$$
g=\left[\left(1+\frac{\lambda M}{7}\right)^{1 / 2}+\frac{4 M}{9 \pi}\right]^{-1 / 2}
$$

which is strictly applicable to a sample with a narrow distribution of molecular weight. Here the parameter $\lambda M$ represents the number of branch units in the molecule. By substituting $g$ in Table $\mathrm{V}$ into eq 8 , we calculated $\lambda M$ and obtained the results given in Table $\mathrm{V}$. It is seen that $\lambda^{-1}$ is essentially independent of $M_{w}$ but decreases with increasing $D C$. This implies that for a given $M_{w}$ the number of branch units increases with incresing $D C$. Since the cyclization reaction involves both intra- and intermolecular reactions, the latter of which leads to branching, the chance of chain branching may increase as the reaction proceeds.

The values of $v$ and $a$ obtained for CBR with $D C$ lower than $50 \%$ are in agreement with the theoretical values $v=0.6$ and $a=0.2 \sim 0.25$ for randomly branched polymers. For $D C$ larger than $50 \%$, especially for the sample CB-E, $v$ is larger than unity and is smaller than 0.2. These values differ considerably from the theoretical values, indicating that the behavior of CB-E in solution cannot be explained by branched structure only. For these high $D C$, a highly compact structure formed by intramolecular crosslinking may appear during the cyclization reaction, as has been pointed out in regard to cyclized polyisoprene. ${ }^{5}$

Acknowledgment. The authors take this opportunity to express their gratitude to Professor M. Kurata of Kyoto University, Dr. K. Ninomiya and Dr. T. Homma of our Research Laboratory for their continuing interest and encouragement throughout this work. They are also grateful to Dr. K. Harada and his group for preparing all CBR samples. Finally, our appreciation goes to Mr. K. Nakahara, Miss S. Kodama and Miss C. Komine for their assistance in the experimental work.

\section{REFERENCFS}

1. Y. Harita, M. Ichikawa, K. Harada, and T. Tsunoda, Polym. Eng. Sci., 17, 372 (1977).

2. V. Bohackova, J. Polacek, and H. Bnoit, J. Chim. Phys., 66, 197 (1969).

3. V. Bohackova, J. Polacek, Z. Grubisic, and H. Bnoit, J. Chim. Phys., 66, 207 (1969).

4. V. Bohackova, E. Fiserova, Z. Grubisic, J. Polacek, and M. Stolka, J. Chim. Phys., 67, 777 (1970).

5. J. Vohlidal, V. Bohackova, and B. Matyska, J. Chim. Phys., 69, 556 (1972).

6. J. Vohlidal, V. Bohackova, and B. Matyska, J. Polym. Sci., Polym. Sympm., No. 42, 907 (1973).

7. M. Abe, K. Hibino, N. Tagata, and T. Homma, Nippon Gomu Kyokaishi, 40, 930 (1967).

8. K. Okita, A. Teramoto, K. Kawahara, and H. Fujita, J. Phys. Chem., 72, 278 (1968).

9. B. H. Zimm, J. Chem. Phys., 16, 1093, 1099 (1948).

10. J. R. Shelton and L-H. Lee, Rubber Chem. Technol., 31, 415 (1958).

11. M. Kurata, "Kobunshi no Bunshisekkei" Vol. 1, Kobunshi Gakkai, Ed, Baifukan, Tokyo, 1972, p 100 (Japanese).

12. B. H. Zimm and W. H. Stockmayer, J. Chem. Phys., 17, 1301 (1949).

13. B. H. Zimm and R. W. Kilb, J. Polym. Sci., 37, 19 (1959).

14. M. Kurata and M. Fukatsu, J. Chem. Phys., 41, 2934 (1964).

15. P. J. Flory, "Principles of Polymer Chemistry," Cornell University Press, Ithaca, New York, 1953.

16. M. Kurata, unpublished calculation. 